\title{
Toxic epidermal necrolysis associated with meibomian gland dysfunction: Report of a case managed by thermal pulsation treatment
}

\author{
Jack V Greiner ${ }^{1,2,3 *}$, Michael E Lindsay ${ }^{1,2}$, Kenneth R Kenyon ${ }^{1,2,3,4}$, Paula J Oliver ${ }^{1,2}$ and Hebah El-Rayess ${ }^{1,2}$ \\ ${ }^{1}$ The Schepens Eye Research Institute, Massachusetts Eye \& Ear, Department of Ophthalmology, Harvard Medical School, Boston, MA, USA \\ ${ }^{2}$ Clinical Eye Research of Boston, Boston and Winchester, MA, USA \\ ${ }^{3}$ Department of Ophthalmology, Tufts University School of Medicine, Boston, MA, USA \\ ${ }^{4}$ Cornea Consultants International, Boston, MA, USA
}

\begin{abstract}
Purpose: To report a case of toxic epidermal necrolysis (TEN) with meibomian gland dysfunction demonstrating improvement in signs and symptoms after a single thermal pulsation system treatment (TPST).

Methods: Baseline signs (meibomian gland assessment [MGA] scores and lipid layer thickness [LLT]) and symptoms (SPEED and OSDI scores) were evaluated in a 41 year old Caucasian female with a 16 years history of dry eye diseases secondary to a TEN episode. The patient was treated with a single 12 -min TPST and re-evaluated at 3,9 , and 36 months post-treatment.

Results: At 3 months post-TPST, both signs and symptoms scores improved relative to baseline and when re-measured at 9 months and 3 years.

Conclusion: This report is the first to suggest the benefit of TPST on improving dry eye signs and symptoms in TEN. Interestingly, the improvement in symptoms as measured by SPEED and OSDI, was paralleled by the improvement in signs (MGA and LLT). Improvements in signs and symptoms were sustained over baseline including the relative decline at 3 years from the optimum improvements post-TPST.
\end{abstract}

\section{Introduction}

Toxic epidermal necrolysis (TEN) is a rare, immune-related disease characterized by skin and mucosal inflammatory lesions, is caused by a severe adverse immune response to medication [1] and is generally considered a more severe variant of Stevens-Johnson syndrome. The incidence of chronic ocular sequelae has been reported to be as high as $90 \%$ of TEN patients [2] with dry eye being the most common [3]. Ocular surface inflammation and ulceration induced by TEN may lead to structural and functional impairment of the eyelid margins including keratinization. Keratinization can secondarily result in closure of the meibomian gland orifices further compromising the tear film lipid layer and resulting in severe dry eye [4]. The degree of impairment to the ocular surface can be so severe leaving limited options for therapeutic intervention.

Although topical lubricant therapy to prevent ocular surface desiccation remains the standard dry eye treatment in TEN cases where meibomian glands have already become dysfunctional, the proven longterm benefit of thermal pulsation system treatment (TPST) for dry eye patients $[5,6]$ suggests TPST may also be especially advantageous.

\section{Report of case}

A 41-year-old Caucasian female with dry eye secondary to an episode of TEN 16 years prior was evaluated. As the ocular history also included right dacryocystorhinostomy 9 years previously with potentially related tear dynamics alteration, only data from the left eye is analyzed. Prior to presentation for TPST, the patient reported using a variety of topical ocular surface lubricants with limited success. Symptoms were assessed using two validated questionnaires- Ocular Surface Disease Index (OSDI) [7] and Standard Patient Evaluation of Eye Dryness (SPEED) [8]. Both questionnaires were employed since the SPEED score provides an assessment of both general and specific dry eye symptoms, whereas the OSDI score is biased toward symptoms that are environmentally and/or behaviorally provoked. On examination, best corrected visual acuity measured 20/20. External exam as expected was notable for the paucity in the number and reduction in the size of the eyelashes, abnormal thickening $(>1 \mathrm{~mm})$ of the eyelid margins, and keratinization with absence of vascular injection and unapparent meibomian gland ostia of the eyelid margins (Figure 1a). Indicative of long-term inflammation secondary to TEN. There was moderate superficial punctate keratitis on the superior quadrant of the cornea after staining with sodium fluorescein and moderate punctate staining of the bulbar conjunctiva after staining with lissamine green. Tearfilm break-up time was $<1$ sec. Meibography was performed with an Oculus Keratograph (Oculus; Arlington, WA) to illustrate the condition of the

${ }^{\star}$ Correspondence to: Dr. Greiner, Schepens Eye Research Institute of Massachusetts Eye \& Ear, W239, 20 Staniford St, Boston, MA 02114, E-mail: Jack_Greiner@meei.harvard.edu

Key words: toxic epidermal necrolysis, thermal pulsation system treatment, lipid layer interferometry, meibomian gland dysfunction

Received: May 23, 2020; Accepted: June 22, 2020; Published: June 26, 2020 
meibomian glands in both the upper and lower eyelids (Figures $1 \mathrm{~b}$ and 1c). The remainder of the examination was unremarkable.

Meibomian gland function was assessed and scored using a meibomian gland evaluator (Tear Science ${ }^{\circledR}$; Morrisville, North Carolina) [9] as follows: The lower eyelid was divided into three equidistant regions (temporal, central and nasal) and five consecutive glands were evaluated for the lower eyelid as previously described [10]. Lipid layer thickness (LLT) was measured by ocular surface interferometry $\left(\right.$ LipiView ${ }^{\circledR}$, Tear Science ${ }^{\circledR}$; Morrisville, North Carolina). Following baseline measurements, a single 12 minute TPST (Lipiflow ${ }^{\circledR}$, Tear Science ${ }^{\circledR}$; Morrisville, North Carolina) was performed. Follow-up examinations occurred at approximately 3,9 , and 36 months post-TPST at which time all measurements were repeated.

At 3, 9 and 36 months post-TPST, signs (MGA and LLT) and symptom (SPEED and OSDI) scores improved over baseline (Table 1). From 3 months to 3 years, symptoms were no longer compelling enough for the patient to seek additional clinical management. Over the entire 3 year interval, the SPEED and OSDI scores decreased $22 \%$ and $14 \%$ from baseline respectively. Following the TPST procedure there was notable reduction in corneal and conjunctival vital staining, The MGA score increased 37\% from baseline and the LLT measurements increased 16\% from baseline. As these measurements are known to vary widely, and as a single case cannot offer statistical analysis, these percent changes were not considered clinically relevant considering absolute measurements (Table 1). However, there was improvement in the patients' wellbeing as measured by both symptom scores. The findings indicated the optimum 3 year improvement in both symptom scores (SPEED and OSDI) occurred at 3 months. This early time-point improvement paralleled the early time-point of improvement in symptoms observed in prior studies [11]. The optimum improvement in signs (MGA and LLT) occurred at 9 months (Table 1). This time line of improvement was consistent with that observed in signs at 9 months in patients with dry eye syndrome [5]. After TPST, although the patient's ab libitum use of topical ocular surface lubricants was not limited, the patient reported marked reduction in use of ocular surface treatments during the 3 year period in contrast to the 16 prior years following the episode of TEN.
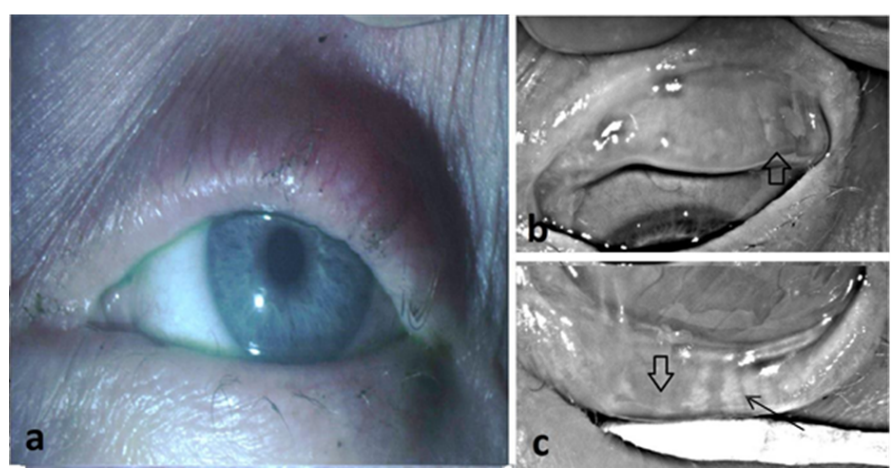

Figure 1. Clinical photographs at initial clinical evaluation demonstrate the characteristics of toxic epidermal necrolysis including dermatologic changes of the periorbital skin, inflammation and keratinization of the eyelid margin, and loss of eyelashes in the upper and lower eyelids (a). High contrast meibography demonstrating severe meibomian gland dropout in the upper (b) and lower (c) eyelids along with remaining meibomian gland remnants (arrowheads). No discernible typical acinar morphology was present in the meibomian gland remnants of the upper lid, nor did gland remnants have any resemblance to a sagitally oriented tubular morphology characteristic of meibomian glands. Few, though more normal appearing, meibomian glands were present in the temporal half of the lower lid and distal portions of some of these glands included the presence of acinar morphology (arrow).
Table 1. Toxic epidermal necrolysis signs and symptom scores at Baseline, 3 months, 9 months, and 36 months post-thermal pulsation system treatment. SPEED, Standard Patient Evaluation of Eye Dryness; OSDI, Ocular Surface Disease Index; MGA, meibomian gland assessment score; LLT, lipid layer thickness (nm)

\begin{tabular}{|c|c|c|c|c|}
\hline & Baseline & 3 months & 9 months & 36 months \\
\hline SPEED [8] & 23 & 8 & 14 & 18 \\
\hline OSDI [7] & 58.33 & 38.64 & 47.9 & 50 \\
\hline MGA [10] & 5 & 13 & 16 & 8 \\
\hline LLT & 53 & 65 & 100 & 63 \\
\hline
\end{tabular}

\section{Comment}

TEN, a debilitating and potentially fatal mucocutaneous disease, is associated with both acute and chronic ocular complications. Typical ophthalmic treatments comprise aggressive topical lubrication, autologous serum eye drops, topical corticosteroids, punctal occlusion, tarsorrhaphy, amniotic membranes, antibiotics, systemic immunosuppressive agents, and symblepharon lysis [2,4]. Remarkably the patient suffered for 16 years relying on traditional lubrication treatments and after undergoing a single TPST, she experienced such sustained asymptomatic improvement that she did not seek further management until 3 years had elapsed. Hence, we conclude that TPST was life-changing for this individual who had previously endured 16 years of dry eye symptoms and for whom use of standard therapy provided little relief. The findings presented herein support the use of TPST in the treatment of TEN.

This study supports the efficacy of TPST even when MGA and meibography scores are unfavorable and patients otherwise are not considered candidates for such thermomechanical intervention with TPST. This report on the use of TPST has implications for TEN patients manifesting severe MGD as evaluated according to the current accepted standard of care protocols as clearly the patient should not have responded according to all the accepted metrics. However, the patient enjoyed the benefits of prolonged symptomatic relief and improvement in signs for up to 3 years after the TPST procedure. This was consistent with prior studies [6] and suggested that regardless of the overt presentation of eyelid margin pathology and presence of meibomian gland drop-out from TEN, the patient may still realize the potential benefit that would otherwise be unexpected. Improvement in signs and symptoms after TPST may even occur after years of being advised that there is nothing else that can be done to improve the condition. The patient in this report was desperate for many years and sought a solution to her dry eye symptoms. Post-TEN a patient with such reduced MGA and meibography demonstrating markedly decreased meibomian glands would not typically be advised to undergo a TPST procedure since this procedure improves meibomian gland function [10-12]. Considering the MGA score and the level of meibomian gland drop-out, the overt clinical appearance may not be an adequate representation of the capability of the eyelid system to respond to TPST short- or long-term. The TPST may thus be overlooked as a possible treatment option. A remarkable duration of improved symptoms is observed in the present case.

\section{References}

1. Hinc-Kasprzyk J, Polak-Krzeminska AG, Ozog-Zabolska I (2015) Toxic epidermal necrolysis. Anaesthesiol Intensive Ther 47: 257-262.

2. Van Zyl L, Carrara H, Lecuona K (2014) Prevalence of chronic ocular complications in Stevens-Johnson syndrome and toxic epidermal necrolysis. Middle East Afr J Ophthalmol 21: 332-335. [Crossref] 
Greiner JV (2020) Toxic epidermal necrolysis associated with meibomian gland dysfunction: Report of a case managed by thermal pulsation treatment

3. Gueudry J, Roujeau JC, Binaghi M, Soubrane G, Muraine M (2009) Risk factors for the development of ocular complications of Stevens-Johnson syndrome and toxic epidermal necrolysis. Arch Dermatol 145: 157-162. [Crossref]

4. Shay E, Kheirkhah A, Liang L, Sheha H, Gregory DG, et al. (2009) Amniotic membrane transplantation as a new therapy for the acute ocular manifestations of Stevens-Johnson syndrome and toxic epidermal necrolysis. Surv Ophthalmol 54: 686-696. [Crossref]

5. Greiner JV (2012) A single LipiFlow ${ }^{\circledR}$ thermal pulsation system treatment improves meibomian gland function and reduces dry eye symptoms for 9 months. Curr Eye Res 37: $272-278$.

6. Greiner, JV (2016) Long-term (3 Year) effects of a single thermal pulsation system treatment on meibomian gland function and dry eye symptoms. Eye Contact Lens 42 99-107.

7. Schiffman RM, Christianson MD, Jacobsen G, Hirsch JD, Reis BL (2000) Reliability and validity of the ocular surface disease index. Arch Ophthalmol 118: 615-621. [Crossref]
8. Hgo W, Situ P, Kier N (2013) Psychometric properties and validation of the standard patient evaluation of dry eye dryness questionnaire. Cornea 32: 12041210. [Crossref]

9. Korb DR, Blackie CA (2008) Meibomian gland diagnostic expressibility: correlation with dry eye symptoms and gland location. Cornea 27: 1142-1147. [Crossref]

10. Lane SS, Dubiner HB, Epstein RJ, Ernest PH, Greiner JV, et al. (2012): A new system, the LipiFlow, for the treatment of meibomian gland dysfunction. Cornea 31: 296-304. [Crossref]

11. Greiner JV (2014) Automatic application of warmth and expression- a breakthrough in physical therapy of dry eye. Acta Ophthalmol 92.

12. Greiner JV (2015) Comparison of efficacy and convenience of warm compresses and eyelid hygiene to thermal pulsation treatment for meibomian gland dysfunction. Am Soc Cataract Refractive Surg. San Diego, CA, April 19, 2015.

Copyright: (C2020 Greiner JV. This is an open-access article distributed under the terms of the Creative Commons Attribution License, which permits unrestricted use, distribution, and reproduction in any medium, provided the original author and source are credited. 